\title{
The Effect of Sample Handling on the Rheological Measurement of Regenerated Silk Fibroin Formic Acid Solution using Parallel Plate Geometry
}

\author{
Hee Jung Cho ${ }^{1}$ and In Chul Um ${ }^{1,2, *}$ \\ ${ }^{1}$ Department of Advanced Organic Materials Science and Engineering, Kyungpook National University, Daegu 702-701, \\ Republic of Korea \\ ${ }^{2}$ Department of Natural Fiber Science, Kyungpook National University, Daegu 702-701, Republic of Korea
}

(Received 03 December 2010; Accepted 20 January 2011)

The effect of sample handling condition on the rheological measurement of regenerated silk fibroin formic acid solution using parallel plate geometry was investigated. In case of loading method, the loading by pouring sample solution resulted in the best reproducibility of rheological measurement. Loading with spoon showed a high variance of viscosity value at low shear rate region $\left(0.01 \sim 1 \mathrm{sec}^{-1}\right)$ while loading with syringe exhibited a low reproducibility of viscosity at high shear region $\left(1 \sim 100 \mathrm{sec}^{-1}\right)$ with a disappearance of shear thinning phenomenon. It was revealed that the sample loading with small extra amount lead to the most reproducible result. The sample loading with the exact amount for the measuring plate resulted in a lack of reproducibility of high shear viscosity, while the loading with large extra volume produced a limited consistency of low shear viscosity. It was turned out that $3 \mathrm{~min}$. of waiting time before measurement was the optimum condition for reliable result. When the waiting time was less than 1 min., the low shear viscosity was obtained with a lack of consistency. On the other hand, the sample solution started drying when the waiting time increased up to $5 \mathrm{~min}$.

Key words: Regenerated silk fibroin, Rheological measurement, Sample handling, Parallel plate geometry

\footnotetext{
*To whom the correspondence addressed Department of Natural Fiber Science, Kyungpook National University, Daegu 702-701, Republic of Korea. Tel: +82-53-950-7757; Fax: +82-53-950-6744; E-mail: icum@knu.ac.kr
}

\section{Introduction}

The silk has been used as an excellent textile material for a long time. For several decades, many researchers have studied the silk to raise its value as a material. As results of those efforts, it was found that silk and regenerated silk had useful properties including a blood compatibility (Sakabe et al., 1989), a good cell attaching and adhesion ability (Minoura et al., 1995), a low inflammatory reaction in body (Santin et al., 1999), etc. With the findings of those properties, recently, many studies have been performed on the application of regenerated silk to biotechnological fields such as tissue engineering scaffold (Baek et al., 2008; Fang et al., 2009; Wang et al., 2006), tympanic membrane (Kim et al., 2010), wound dressing (Schneider et al., 2009), etc.

In order to apply the regenerated silk to those applications, raw silk cocoon should be fabricated to fiber or film form. The raw silk cocoon is dissolved in solvent to prepare silk solution, and then the silk solution was transformed to fiber or film form by wet spinning, electrospinning, or film casting process. During the spinning process, the rheology of silk solution is important in fiber fabrication because it strongly affects wet- and electrospinning process.

The rheology is a science related to the deformation of material by force. The rheology dominates the wet and electro-spinnability since the dope solution should be deformed properly to micro or nanosized fiber form by external forces such as pressure and electric force. In case of electrospinning, if the solution viscosity is too high, the electric force can not overcome the viscosity resulting in a failure of spinning. If the solution viscosity is too low, solution is deformed to discontinuous form leading to a 
bead formation rather than a fiber formation (Deitzel et al., 2001; Sachiko et al., 2003). Therefore, it can be said that there is a proper viscosity range for nanofiber fabrication. In the viscosity range, the viscosity determines the fiber diameter of spun fiber. That is, the diameter of fiber increased with an increase of dope solution viscosity (Gupta et al., 2005; Sill et al., 2008). In also wet spinning and film casting process, similar principle is applied. Based on the importance of rheology of dope solution, it has been measured and studied in the research regarding wet- and electro- spinning process (Mckee et al., 2004). Recently, silk formic acid dope solution has been utilized extensively in wet- and electro-spinning because of its good spinnability (Cho et al., 2010; Jeong et al., 2005; Ko et al., 2010; Min et al., 2004; Um et al., 2004). However, the viscosity of silk formic acid solution is relatively low and the solution is too sensitive to an external force. Especially, it seems that the force history strongly influences the rheological measurement of silk formic acid solution. As a result of that, the reproducibility of rheological measurement of silk formic acid solution is relatively low leading to a low reliability of result.

Therefore, in this study, the effect of sample handling condition on the rheological measurement of silk formic acid solution using parallel plate geometry to find out the optimum sample handling condition to have reproducible rheological measurement.

\section{Materials and Methods}

\section{Preparation}

Bombyx mori cocoons were degummed with a sodium oleate $0.3 \%$ (o.w.f.) and sodium carbonate $0.2 \%$ (o.w.f.) solution at a boiling temperature for 1 hour, and then rinsed thoroughly in warm distilled water and dried to obtain a silk fibroin. The silk fibroin was dissolved in 9.3 $\mathrm{M} \mathrm{LiBr}$ aqueous solution at room temperature for 24 hours. The aqueous silk fibroin solutions were obtained through the dialysis of the dissolved silk fibroin solutions in a cellulose tube (molecular cutoff $=12,000$ 14,000 ) against a circulating pure water at room temperature for 4 days. The solution was then dried at $60^{\circ} \mathrm{C}$ and ground to obtain regenerated silk fibroin powder. The regenerated silk fibroin films were dissolved in $98 \%$ formic acid to produce $12 \%(\mathrm{w} / \mathrm{w})$ silk fibroin formic acid solution. The solution was filtered through a nonwoven fabric twice to remove insoluble particles to use in the rheological measurement.

\section{Sample handling}

Various sample handling factors were tested to find out their effect on the rheological measurement of silk fibroin formic acid solution. First of all, three loading methods, 1) pouring solution, 2) loading with spoon, and 3) loading with syringe, were tried. Second, the amount of sample loading was controlled from $1.8 \mathrm{~g}$ to $2.95 \mathrm{~g}$. Last, the waiting time between the finish of sample loading and the measurement start was varied from $0 \mathrm{~min}$. to $5 \mathrm{~min}$.

\section{Rheological measurement}

The rheology of regenerated silk fibroin formic acid solution was measured by rheometer (MARS III, Hakke, Germany). A parallel geometry was used and the radius of plate was $35 \mathrm{~mm}$. Shear viscosity was measured in the function of shear rate $\left(0.01 \sim 100 \mathrm{sec}^{-1}\right)$. The rheological measurement was performed at $25^{\circ} \mathrm{C}$.

\section{Results and Discussion}

\section{The effect of loading method}

The history of force applied to sample before the rheological measurement strongly influences the result of measurement. When the solution sample is loaded, it experiences deformation by an external force. For instance, if the sample is pouring on the measurement plate, the gravity will deform the solution state of sample. In also sampling with spoon, the external force will deform the molecular state in solution. In case of sampling with syringe, though the amount of sample loading can be controlled precisely, a high shear force can be applied to solution.

All those loading methods can not prevent the sample solution from being deformed by the external force. However, it is important to know which method can deform solution sample less and produce a reproducible result. Therefore, the effect of loading method on the rheological measurement of regenerated silk fibroin acid solution was investigated.

The regenerated silk fibroin formic acid solution was loaded with three different methods; loading by pouring, spoon, and syringe. And then, the steady flow test was performed $4 \sim 5$ times using the three loading methods. The test results were presented in Fig. 1.

The sample loaded by pouring (Fig. 1(a)) showed relatively reproducible result. The sample showed a shear thinning implying a molecular entanglement of silk fibroin in formic acid. This means the molecular entanglement of silk was not disrupted by the gravity. However, when the sample was loaded with spoon (Fig. 1(b)), the viscosity variation at low shear rate region $\left(0.01 \sim 1 \mathrm{sec}^{-1}\right)$ was high. In case of syringe loading, the shear viscosity decreased much at even $1.0 \mathrm{sec}^{-1}$ of shear rate, resulting in a disappearance of shear thinning. Furthermore, the vari- 


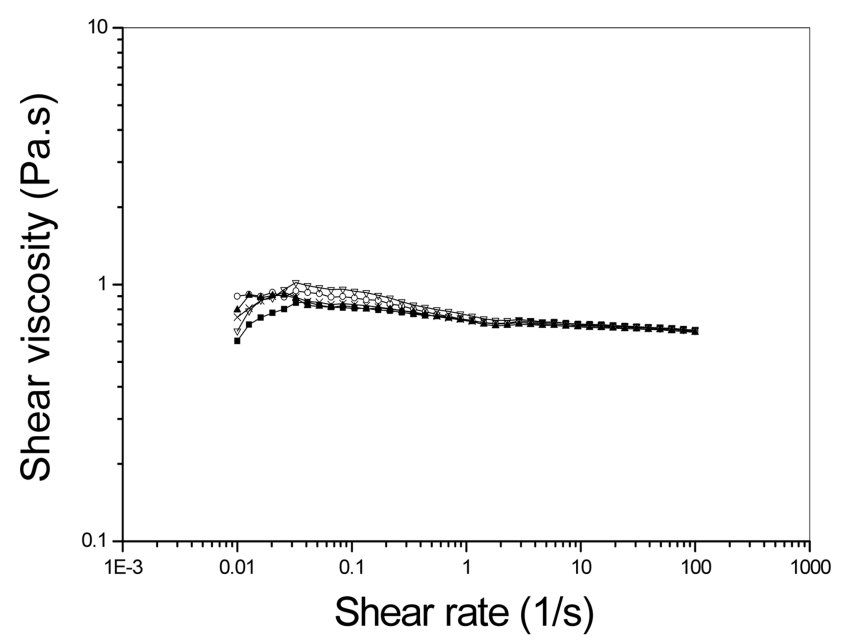

(a)

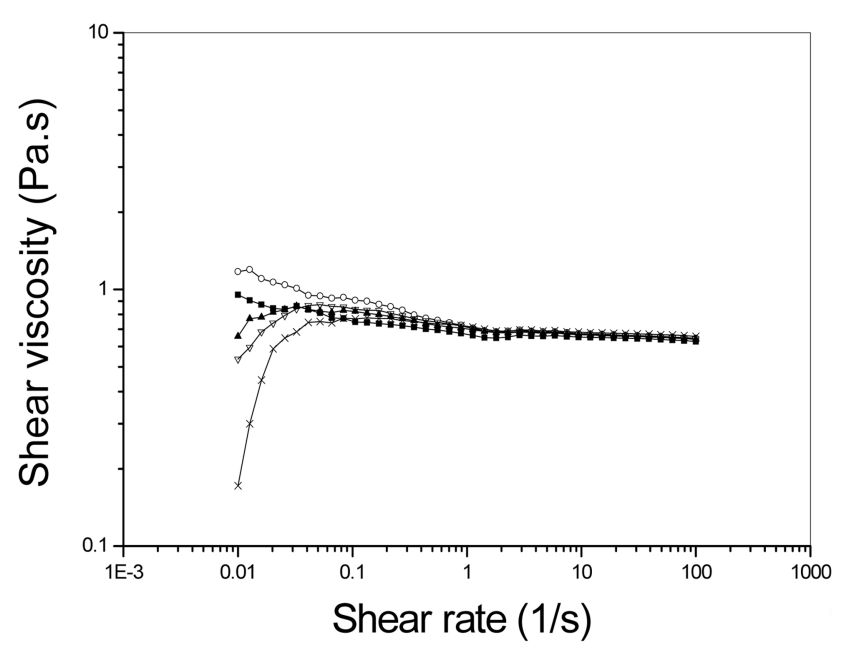

(b)

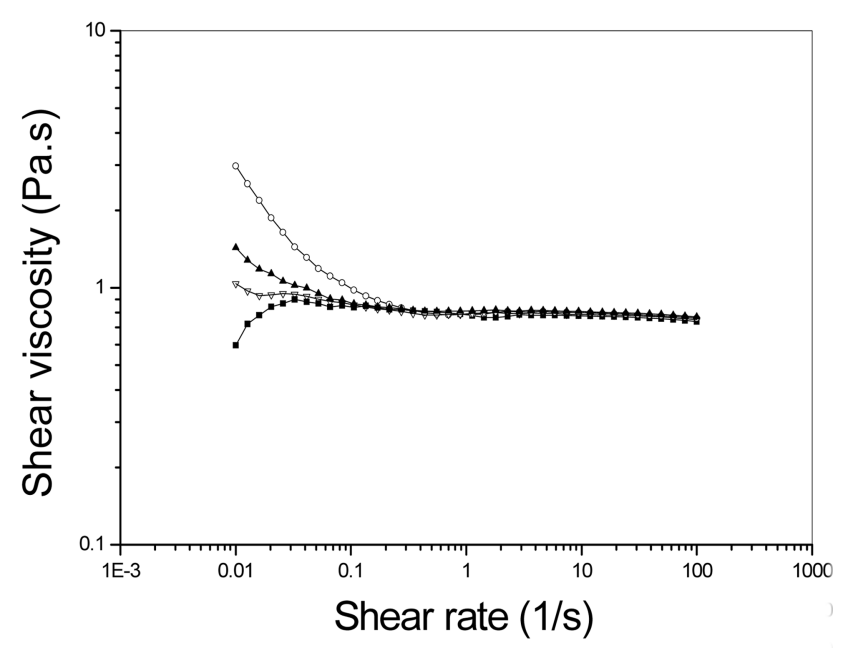

(c)

Fig. 1. The effect of sample loading method on the steady flow test result of $12 \%(\mathrm{w} / \mathrm{w})$ regenerated silk fibroin formic acid solution; (a) pouring, (b) spoon and (c) syringe. ation of viscosity value at low shear region was high.

From the results in Fig. 1, the variation of viscosity value at low shear region is a key point to have reproducible rheology measuring data considering the viscosity at high shear did not change by the different loading method. It is natural considering the effect of shear force on the molecular entanglement in solution. Most polymer molecules are entangled in concentrated solution. As a result of the entanglement, a high viscosity at low shear rate region appeared in steady flow test. However, as the shear rate increased, the entangled molecules became unentangled resulting in a decrease of viscosity. Therefore, when small or steady force is applied to sample solution, the molecular entanglement keeps unchanged or changes a little bit.

It seems that the loading by pouring is the proper loading method among the tested. The fact that pouring solution on the plate produced reproducible results, might be due to that it is the simplest way. In case of the loading with spoon, when the solution on the spoon is moving to measurement plate, an irregular force can be applied to the solution by each experimenter resulting in an irregular disruption of molecular entanglement of silk. As a result of that, the variation of viscosity at low shear region can increase. Though the amount of sample can be controlled easily, the loading with syringe was turned out to be the worst loading way. The high variation of viscosity at low shear might be due to the high shear induced by pushing syringe. That is, when the sample solution was squeezed out of the syringe, a high shear force can be applied to sample. Therefore, the disappearance of shear thinning by the syringe loading might be attributed to that the molecular entanglement was disrupted severely by the high shear force.

\section{The effect of amount of sample loading}

The viscosity is calculated by the ratio of shear stress and shear rate which are measured in the rheometer. In case of shear stress, it is determined by the ratio of shear force and dimension. Therefore, if the sample solution does not cover the measuring plate, the measured force will be reduced resulting in a decrease of shear stress and shear viscosity. On the other hand, if the amount of sample loading is too much, the extra amount of sample will restrict the deformation of sample in measuring plate leading to a decrease of shear rate and an increase of shear viscosity. With those reasons, it is necessary to find a proper amount of sample loading for reproducible measuring data.

In this study, three different amount of sample were tested. Fig. 2. exhibited the feature of loaded solutions between the parallel plate. When $1.8 \mathrm{~g}$ sample solution (Fig. 2(a)) was used, the sample solution covered the plate exactly. In case of $2.2 \mathrm{~g}$ sample (Fig. 2 (b)), there was 


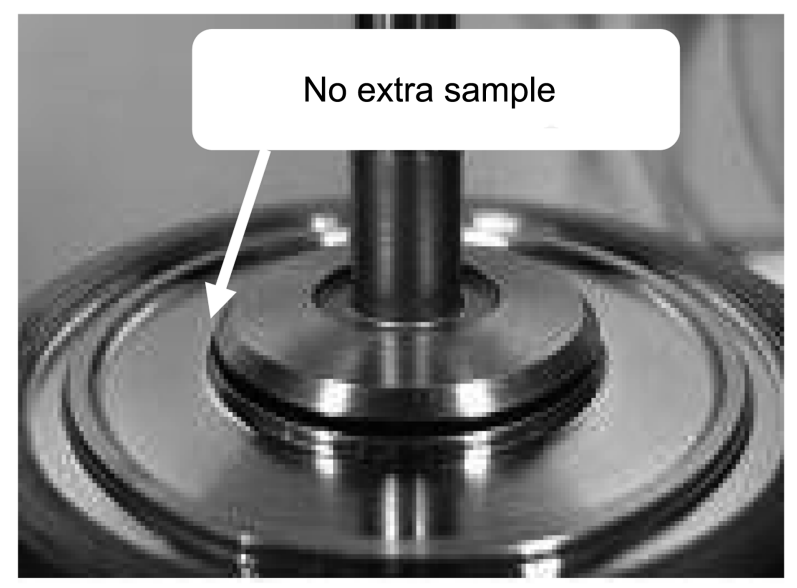

(a)

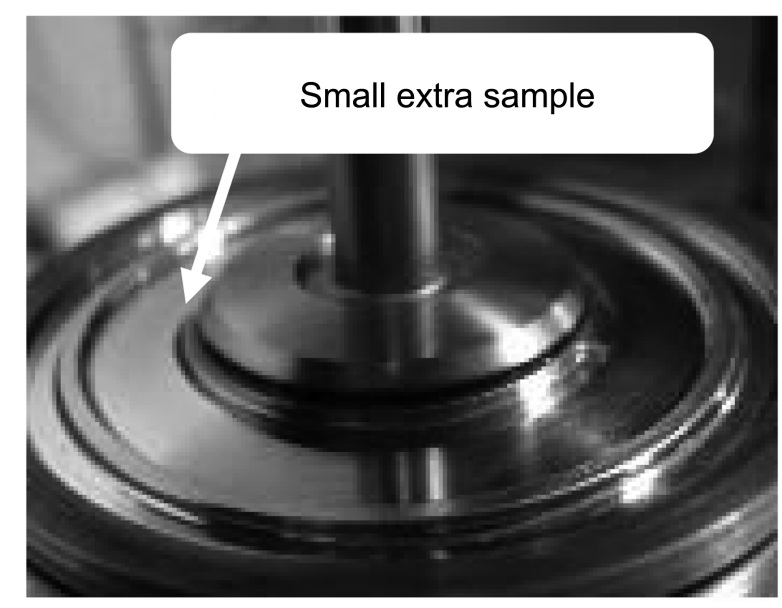

(b)

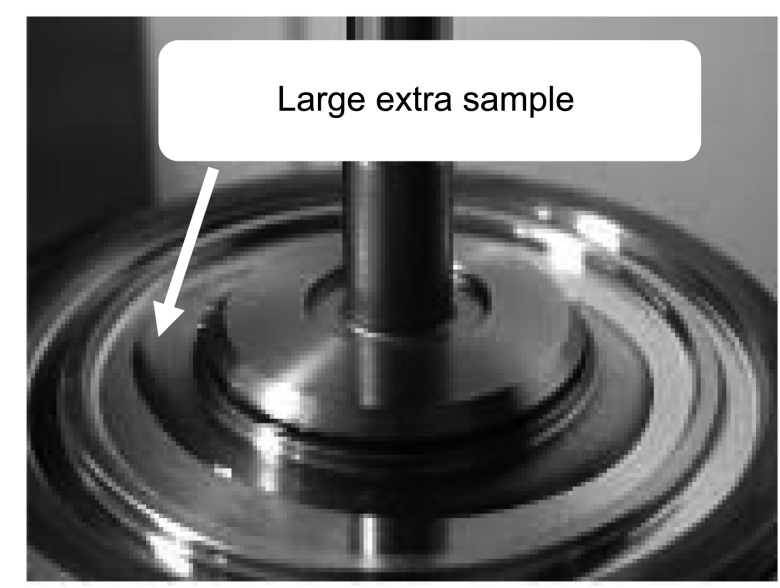

(c)

Fig. 2. The photographs of loaded silk solution with various sample amounts; (a) $1.8 \mathrm{~g}$, (b) $2.2 \mathrm{~g}$ and (c) $2.95 \mathrm{~g}$.

small extra solution while $2.95 \mathrm{~g}$ solution resulted in large extra solution sample (Fig. 2 (c)).

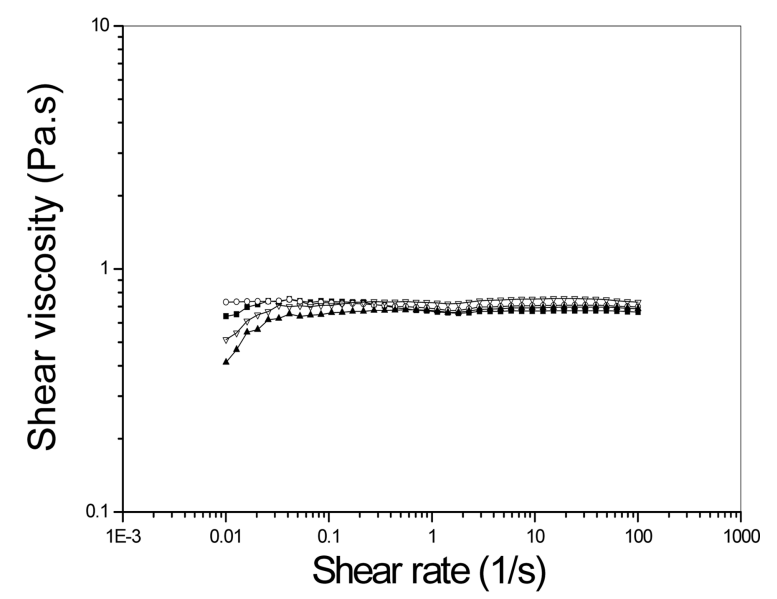

(a)

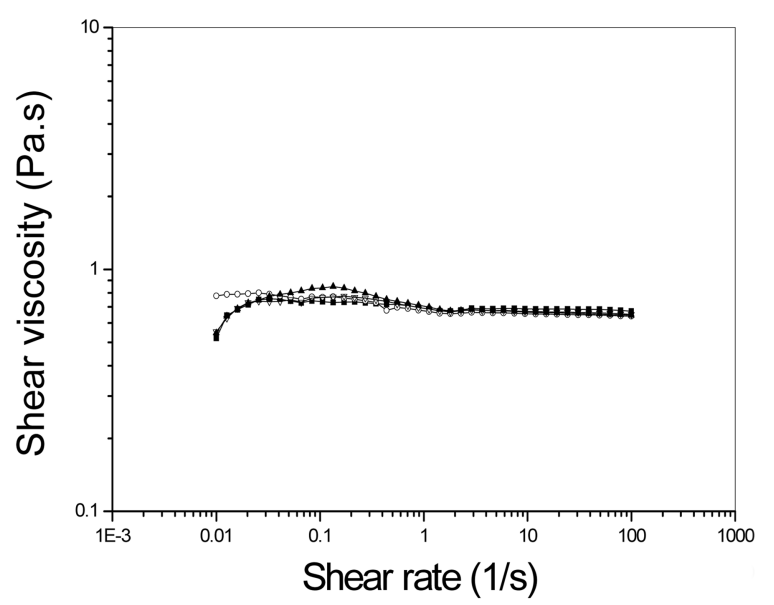

(b)

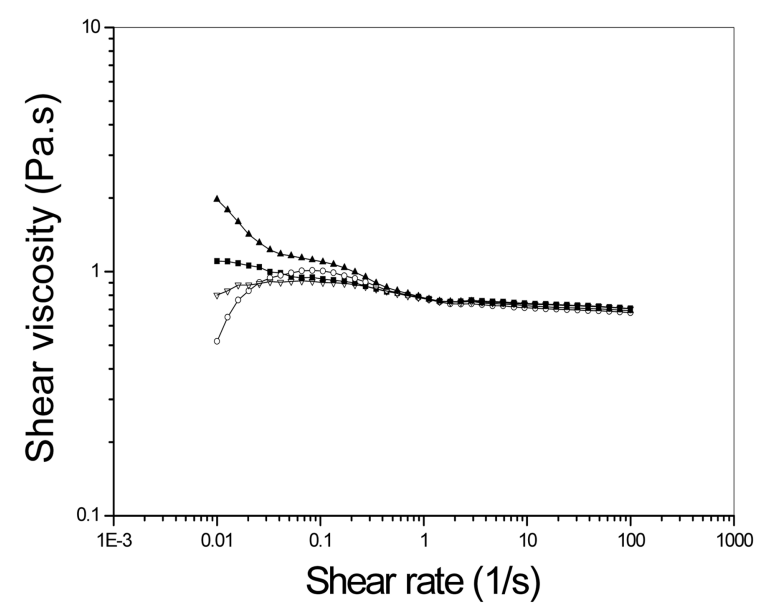

(c)

Fig. 3. The effect of sample amount on the steady flow test result of $12 \%(\mathrm{w} / \mathrm{w})$ regenerated silk fibroin formic acid solution; (a) $1.8 \mathrm{~g}$, (b) $2.2 \mathrm{~g}$ and (c) $2.95 \mathrm{~g}$.

Fig. 3. showed the effect of the amount of sample loading on the relationship between shear viscosity and shear 


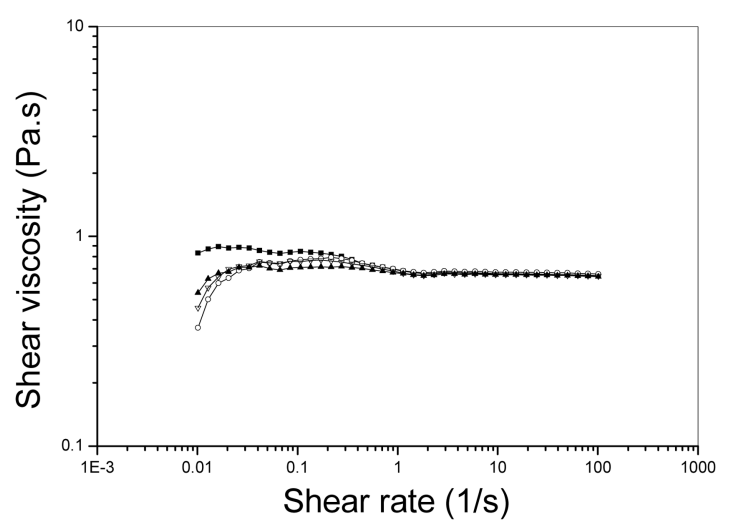

(a)

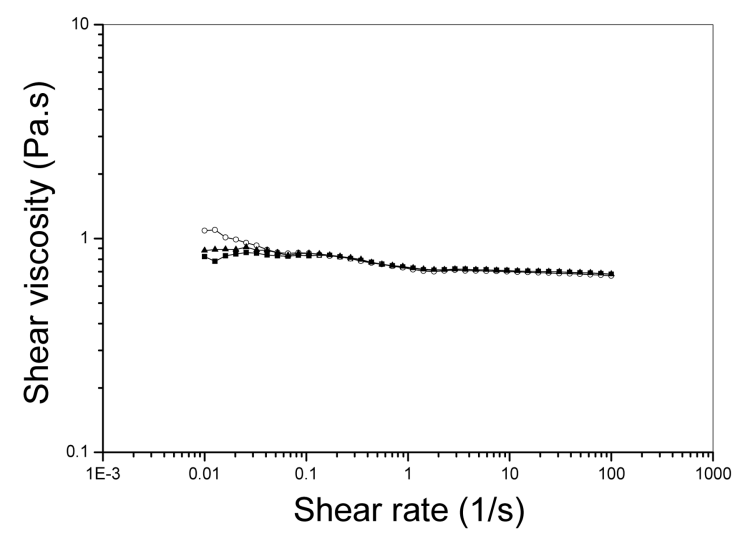

(c)

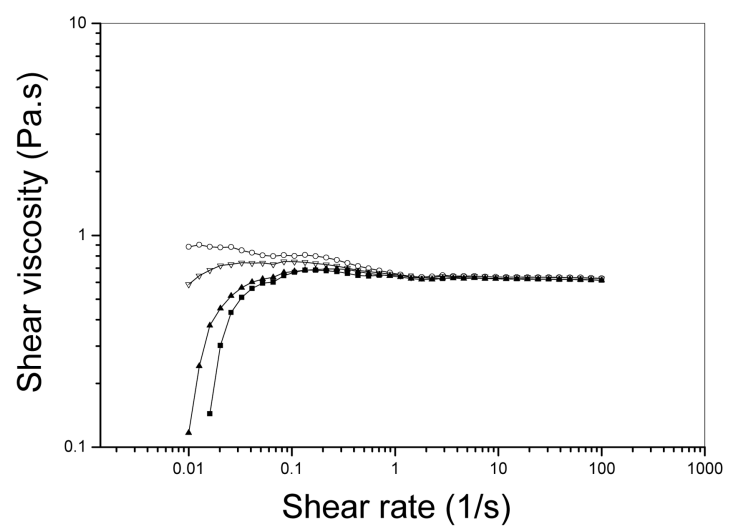

(b)

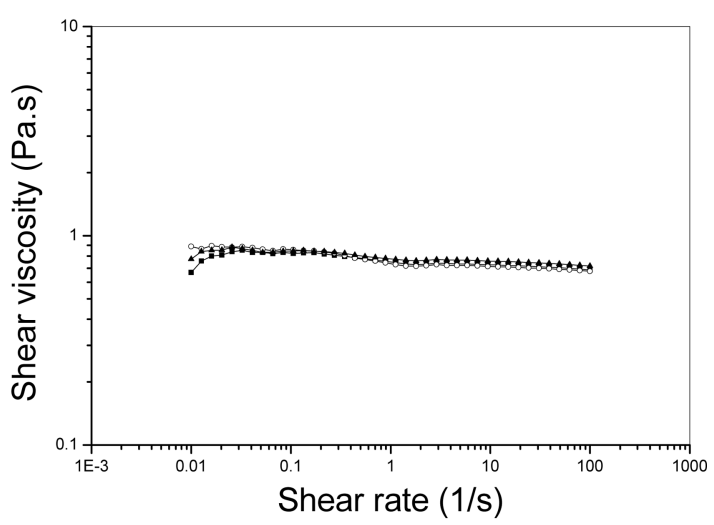

(d)

Fig. 4. The effect of the waiting time on the steady flow test result of $12 \%$ (w/w) regenerated silk fibroin formic acid solution; (a) 0 min., (b) 1 min., (c) 3 min. and (d) 5 min.

rate. In case of $1.8 \mathrm{~g}$ sample (Fig. 3(a)), though the variation of viscosity at low shear region $\left(0.01 \sim 1 \mathrm{sec}^{-1}\right)$ was not high, there was also small variation of viscosity at high shear region $\left(1 \sim 100 \mathrm{sec}^{-1}\right)$. The variation of viscosity at high shear might be due to a deviation of some sample from measuring plate by centrifugal force during high shear force region. That is, as the sample loss is occured at high shear region, the shear stress is decreased resulting in a shear viscosity reduction.

On the other hand, $2.95 \mathrm{~g}$ solution showed a high variation of viscosity value at low shear region, while it exhibited little variation of viscosity at high shear region. The reproducible viscosity at high shear might be attributed to the absence of sample loss by the centrifugal force because the amount of sample was much. It seemed that the variation of viscosity at low shear was due to that the extra solution suppressed the deformation of sample. $2.2 \mathrm{~g}$ solution showed the best reproducibility of result among the samples. This sample with small amount of extra solution did not have a problem of sample loss during the high shear region. Also, the small extra solution did not restrict the deformation of sample solution much. Therefore, it seems a small extra solution is needed to have the reproducibility of measuring data.

\section{The effect of waiting time before measurement}

Even if the sample loading is conducted carefully, the deformation of solution sample can take plate. Therefore, it is necessary to wait a time for silk fibroin molecules to recover original molecular state from the disentanglement induced by the sample loading. Based on this background, the effect of waiting time on the rheological measurement of silk fibroin formic acid solution was investigated to find out the optimum waiting time.

Fig. 4. displayed the steady flow test result of regenerated silk fibroin formic acid solution with different waiting time before measurement. As can be seen, 0 and $1 \mathrm{~min}$. (Fig. 4(a) and (b)) showed a high variation of viscosity result at low shear region implying that less than $1 \mathrm{~min}$. is not enough for silk molecules to recover their original state. On the other hand, $3 \mathrm{~min}$. of waiting time produced a highly reproducible result as can be seen in Fig. 4(c). This indicated that at least 
3 min. of waiting time was needed to recover silk solution state. When the waiting time was increased up to $5 \mathrm{~min}$., there was a little variation of viscosity at both low and high shear region. This is due to fact that the exposed solution at a fringe of measurement region started drying at $5 \mathrm{~min}$. of waiting time. That is, a thin film was formed on the sample solution and this prevented a precise rheological measurement. Based on those results, it can be concluded that $3 \mathrm{~min}$. of waiting time is the optimum waiting time for regenerated silk fibroin formic acid solution.

In this study, three sample handling factors, 1) loading method, 2) amount of sample loading, and 3) waiting time before measurement, were tested to find out their effects on the rheological measurement of silk fibroin formic acid solution and to find the optimum sample handling condition for the reproducible measuring data. All factors tested here were turned out to have significant effects on the rheological measurement of silk formic acid solution. Therefore, when the rheology of silk fibroin formic acid solution is measured using rheometer, those handling conditions should be considered carefully to obtain reliable result. In the present study, 1) pouring as a loading method, 2) sample loading with small extra solution, and 3) $3 \mathrm{~min}$. of waiting time were elucidated as the optimum conditions for $12 \%$ silk fibroin formic acid solution. However, different type of silk solution, such as concentrated silk aqueous solution, and silk solution with different sericin content should be also examined to find out the operation factors influencing rheological measurement. Though parallel plate geometry was used in this study, it is known that a cone and plate geometry can provide more precise result. Therefore, when the rheometer with cone and plate geometry is used in silk solution, it is also necessary to investigate the effect of sample handling on the rheological measurement in the next study.

\section{Acknowledgement}

This research was supported by Technology Development Program for 'Agriculture and Forestry', Ministry for Food, Agriculture, Forestry and Fisheries, Republic of Korea, 2010.

\section{References}

Baek HS, Park YH, Ki CS, Park J, Rah DK (2008) Enhanced chondrogenic responses of articular chondrocytes onto porous silk fibroin scaffolds treated with microwave-induced argon plasma. Surf Coat Tech 202, 5794-5797.

Cho HJ, Um IC (2010) The effect of dissolution condition on the yield, molecular weight, and wet- and electro-spinnability of regenerated silk fibroins prepared by $\mathrm{LiBr}$ aqueous solution. Int J Indust Entomol 20, 99-105.

Deitzel JM, Kleinmeyer J, Harris D, Beck NC (2001) The effect of processing variables on the morphology of electrospun nanofibers and textiles. Polymer 42, 261-272.

Fang Q, Chen D, Yang Z, Li M (2009) In vitro and in vivo research on using Antheraea pernyi silk fibroin as tissue engineering tendon scaffolds. Mater Sci Eng 29, 1527-1534.

Gupta P, Elkins C, Long TE, Wilkes GL (2005) Electrospinning of linear homopolymers of poly(methyl methacrylate): exploring relationships between fiber formation, viscosity, molecular weight and concentration in a good solvent. Polymer 46, 4799-4810.

Jeong L, Park WH (2005) Fabrication and applications of silk Fibroin nanofibers. Polym Sci Technol 16, 556-567.

Kim J, Kim CH, Park CH, SeoJ, Kweon HY, Kang SW, Lee KG (2010) Comparison of methods for the repair of acute tympanic membrane perforations: Silk patch vs. paper patch. Wound Rep Reg 18, 132-138.

Ko JS, Lee KH, Bae DG, Um IC (2010) Miscibility, structural characteristics, and thermal behavior of wet spun regenerated silk fibroin/Nylon 6 blend filaments. Fib Polym 11, 14-20.

Mckee MG, Wilkes GL, Colby RH, Long TE (2004) Correlations of solution rheology with electrospun fiber formation of linear and branched polyesters. Macromolecules 37, 1760-1767.

Min B, Lee G, Kim SH, Nam YS, Lee TS, Park WH (2004) Electrospinning of silk fibroin nanofibers and its effect on the adhesion and spreading of normal human keratinocytes and fibroblasts in vitro. Biomaterials 25, 1289-1297.

Minoura N, Aiba S, Gotoh Y, Tsukada M, Imai Y (1995) Attachment and growth of cultured fibroblast cells on silk protein matrices. J. Biomed Mater Res 29, 1215-1221.

Sachiko S, Gandhi M, Ayutsede J, Micklus M, Ko F (2003) Regeneration of Bombyx mori silk by electrospinning - part 1: processing parameters and geometric properties. Polymer 44, 5721-5727.

Sakabe H, Ito H, Miyamoto T, Noishiki Y, Ha WS (1989) In vivo blood compatibility of regenerated silk fibroin. Sen-i Gakkaish 45, 487-490.

Santin M, Motta A, Freddi G, Cannas M (1999) In vitro evaluation of the inflammatory potential of the silk fibroin. $J$ Biomed Mater Res 46, 382-389.

Schneider A, Wang XY, Kaplan DL, Garlick JA, Egles C (2009) Biofunctionalized electrospun silk mats as a topical bioactive dressing for accelerated wound healing. Acta Biomaterialia 5, 2570-2578.

Sill TJ, Recum HA (2008) Electrospinning: applications in drug delivery and tissue engineering. Biomaterials 29, 2989-2006.

Um IC, Kweon HY, Lee KG, Ihm DW, Lee J, Park YH (2004) Wet spinning of silk polymer: I. Effect of coagulation conditions on the morphological feature of filament. Int $\mathrm{J}$ Biol Macromol 34, 89-105.

Wang Y, Blasioli DJ, Kim H, Kim HS, Kaplana DL (2006) Cartilage tissue engineering with silk scaffolds and human articular chondrocytes. Biomaterials 27, 4434-4442. 